\title{
Emergency department and hospital crowding: causes, consequences, and cures
}

\author{
Peter McKenna, Samita M Heslin, Peter Viccellio, William K Mallon, \\ Cristina Hernandez, Eric J Morley \\ Department of Emergency Medicine, Stony Brook University Hospital, Stony Brook, NY, USA
}

Overcrowding with associated delays in patient care is a problem faced by emergency departments (EDs) worldwide. ED overcrowding can be the result of poor ED department design and prolonged throughput due to staffing, ancillary service performance, and flow processes. As such, the problem may be addressed by process improvements within the ED. A broad body of literature demonstrates that ED overcrowding can be a function of hospital capacity rather than an ED specific issue. Lack of institutional capacity leads to boarding in the ED with resultant ED crowding. This is a problem not solvable by the ED and must be addressed as an institution-wide problem. This paper discusses the causes of ED overcrowding, provides a brief overview of the drastic consequences, and discusses possible cures that have been successfully implemented.

Keywords Crowding; Emergency service, hospital; Patient safety

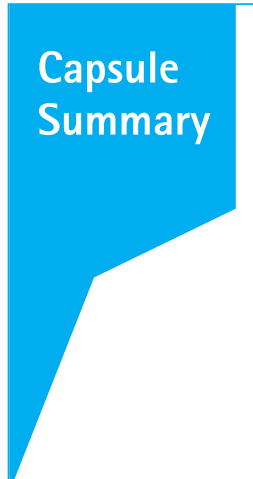

What is already known

A growing body of literature demonstrates that emergency department overcrowding can be a function of hospital capacity rather than an emergency department specific issue.

What is new in the current study

This paper discusses the causes of emergency department overcrowding, provides a brief overview of the drastic consequences, and discusses possible cures that have been successfully implemented.
elSSN: 2383-4625

Received: 7 April 2018

Revised: 29 June 2018

Accepted: 4 July 2018

Correspondence to: Samita M Heslin Department of Emergency Medicine, Stony Brook University Hospital, 101 Nicolls Road, HSC Level 4-080, Stony Brook, NY 11794, USA

E-mail:Samita.Heslin@

StonyBrookMedicine.edu ORCID

http://orcid.org/0000-0002-7837-8834

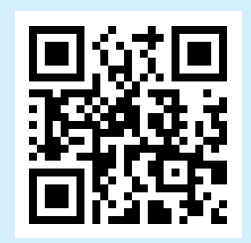

How to cite this article:

McKenna P, Heslin SM, Viccellio P, Mallon WK, Hernandez C, Morley E. Emergency department and hospital crowding: causes, consequences, and cures. Clin Exp Emerg Med 2019;6(3):189-195.

This is an Open Access article distributed under the terms of the Creative Commons Attribution Non-Commercial License (http:// creativecommons.org/licenses/by-nc/4.0/). 


\section{INTRODUCTION}

In the emergency department (ED), multiple medical services, providers, and ancillary staff must work together to triage, diagnose, and treat medical problems ranging from the benign to the life threatening. Since its inception, the ED has been negatively affected by overcrowding, defined by the American College of Emergency Physicians as a situation in which "...the identified need for emergency services exceeds available resources for patient care in the ED, hospital, or both." ${ }^{11}$ Countless studies and interventions have been targeted at improving flow through the $E D$, especially in high volume EDs, where overcrowding can lead to patient safety issues. Efforts to improve patient flow have often met with very limited success. Why? Although the problem of overcrowding is most visible in the ED, it is strongly associated with boarding of admitted patients due to inadequate hospital capacity. This problem cannot be overcome by process improvements limited to the ED nor by diverting low acuity patients, since they do not address the underlying problem caused by boarding. ${ }^{2,3}$ Boarding reflects an institution-wide lack of capacity, thus suggesting that the better term is hospital overcrowding.

The ED is the gateway to the hospital. In the United States, a total of 131 million ED visits resulted in 14.5 million admissions $(11 \%)$ in 2012 , of which $13 \%$ were admitted to a critical care unit. ${ }^{4}$ In South Korea, of the 4.97 million ED visits recorded in the 2012 National Emergency Department Information System, 995,326 resulted in admission (20\%), of which 14\% were admitted to an intensive care unit. ${ }^{5}$ The scope of practice in Emergency Medicine is expanding to include prolonged observational stays, more complex diagnostic workups, and increased critical care and intensive care within the ED. As this occurs, the strain of an overcrowded department is becoming more evident each day as patients spend more and more hours in the ED receiving definitive care and diagnostic workups for conditions that were previously the domain of the inpatient services.

\section{CONSEQUENCES}

ED overcrowding has true costs and has real consequences, in both patient care and the cost of medicine. Overcrowding causes delays in care for all patients, including the critically ill, 10\% of whom wait more than one hour to see a physician according to the Centers for Disease Control and Prevention. ${ }^{6}$ A 2009 study found that serious complications increased 3-5 fold in acute coronary syndrome patients who presented at times of overcrowding. A 2012 study showed that ED crowding increased 28-day mortality rate in community acquired pneumonia patients. ${ }^{8} \mathrm{ED}$ boarding has been demonstrated to increase total hospital length of stay (LOS) by at least one day, with the longest boarders having increased LOS of 3 days. ${ }^{9-18}$ Boarding increases the number of patients who leave without being seen, including those with serious illnesses. ${ }^{19,20}$ Boarding also increases the incidence of medical errors and reduces the quality of care that can be provided by overwhelmed staff. ${ }^{21-23}$ This reduced quality of care has been demonstrated in delays in care of asthmatics, ${ }_{1}^{24}$ patients requiring antibiotics for pneumonia, ${ }_{1}^{25-27}$ and adults needing pain relief. ${ }^{28,29}$ ED boarding of admitted patients causes inefficiency within the department, leading to lost revenues from walkouts and diversions, increased LOS and loss of revenues from emergency medicine admissions. ${ }^{14,30-33}$ Most importantly, boarding in the ED is directly related to increases in 10- and 30-day mortality. ${ }^{34-36}$ Reducing boarding and improving hospital capacity leads to lower overall mortality rates. ${ }^{37,38}$

\section{INNEFFECTIVE INTERVENTIONS}

With the major cause of ED overcrowding identified as a bottleneck of admitted patients waiting for an inpatient bed and a lack of hospital capacity, it is clear why the interventions targeted at ED flow have been only minimally effective. Diverting non-urgent patients has shown little difference in terms of flow, and is a patient safety issue as many "non-urgent" patients will eventually require hospital admission. ${ }^{39,40}$ Expanding ED capacity does little to reduce time to admission but rather increases the capacity for boarded patients, further straining an already overwhelmed staff. ${ }^{41-43}$ While additional staff from the inpatient unit can help relieve the strain on the ED nursing staff, boarded patients still create space constraints limiting the capacity of the ED to see new patients. ${ }^{44}$

\section{CURES}

In order to truly address the problem of ED overcrowding, we must address the problem of hospital capacity. Viccellio et al..$^{45}$ identified four major areas that must be targeted to improve hospital capacity and decrease ED boarding: smoothing of elective admissions, early discharge, weekend discharge, and full capacity plans.

\section{Smoothing elective admissions}

While the variability of emergency medicine admissions is uncontrollable, studies over time have shown that it is highly predictable based on time, season, and epidemiology, ${ }_{1}^{4-48}$ resulting in a smooth pattern of admissions without a great deal of variability from day to day. The remainder of hospital admissions are elec- 
tive scheduled admissions which, historically, have been scheduled early in the week to suit the needs of specialty practices that tend to follow a more regular schedule of working hours. In fact, studies from The Journal of Hospital Medicine and the Annals of Surgery have demonstrated that elective surgical admissions can vary by a factor of 3 between weekdays. ${ }^{49,50}$ These large variations in elective admissions create a bottleneck early in the week, resulting in elective patients competing directly with ED patients for intensive care unit (ICU) and floor beds. Given the spikes in elective admissions early in the week, "smoothing" these admissions and evenly distributing them across the week would improve hospital bed capacity and, therefore, improve ED flow by decreasing boarding. ${ }^{51-54}$

In 2003, Boston Medical Center, with a volume of 120,000 ED visits was faced with overcrowding, boarding, and diversion. Their overcrowding assessment found that the variability in elective admissions drove ED boarding. Boston Medical Center then smoothed admissions across the week for each destination-step down unit vs. ICU vs. regular floor bed-to maximize operating room efficiency. ${ }^{45,55}$ The results were impressive: ambulance diversion decreased; LOS decreased by 45 minutes; waiting room time decreased by 20 minutes; cancelled procedures dropped by 99.5\%; and ICU and step down unit variability decreased by $55 \%$, all while seeing more patients than the year prior. ${ }^{45,55}$

\section{Early discharge}

As the volume within the ED increases throughout the course of the day, the volume of patients being admitted to the hospital increases. Without the early discharge of inpatients, newly admitted ED patients become ED boarders. A focus on early discharge before noon has been demonstrated to improve the flow of the ED by decompressing the number of ED boarders prior to the time the ED is at its busiest. ${ }^{56-58}$ In 2011, NYU-Langone Medical Center was faced with increasing patient volume and patient acuity. Metrics identified that admitted patients arriving on the floors after 1 p.m. remained in the hospital 0.6 days longer than those that arrived before 1 p.m. Throughout 2011, less than 10\% of patients were discharged before 12 p.m., preventing admitted ED patients from moving to the floor. This often delayed initiation of necessary tests and interventions such as social services, case management, and consults, leading to an increased length of stay. ${ }^{45,59,60}$ NYU-Langone Medical Center launched the Discharge Before Noon Challenge (DBN) in March of 2012. Multiple causes of late discharge were identified, including patient desire to stay for meals, wait for transportation, or discuss final recommendations with their care team. The initiative involved all healthcare workers within the hospital, from the chief of medicine and nurs- ing staff to the clerks and transporters. It was made clear to the health care team that discharging patients earlier in the day was in their best interest, allowing patients time during the day of discharge to establish home care, obtain durable medical equipment, and obtain prescription medications. In order to achieve early discharges, major institutional changes were implemented: health care team members' roles were clarified; checklists were designed; and geographical wards were created to streamline communication between nurses and physicians. ${ }^{45,60}$ With geographic wards, the health care team functioned as one team in one area, allowing for interdisciplinary rounds involving physicians, nurses, social workers, and case managers. Patients were notified of their plan of care and those patients identified as possible discharges the next morning were prioritized for testing and consults. Three months after the DBN initiative began, the discharge before noon rate increased from 5\% to 30\%. Over five years, that rate increased to $42 \%$. The DBN initiative did not increase readmission rates, and decreased the observed to expected LOS by 0.8 days. $^{45}$

\section{Weekend discharge}

Further variability occurs during the weekends, when the number of discharges is almost 50\% less than the number of weekday discharges. The NY SPARCS (Statewide Planning and Research Cooperative System) data shows that LOS is 1.22 days shorter for medicine patients discharged on a Saturday, compared to patients discharged on Monday. Similarly, LOS increases to 2 days for surgical patients. Thus, increasing weekend discharges can increase inpatient hospital capacity, decrease ED boarding, and decrease overall hospital LOS. ${ }^{61}$ The impact of increasing weekend discharges is best demonstrated by the success of the Montefiore Medical Center (MMC) in creating an institutional focus on weekend discharges. A hospital that operates at near $100 \%$ capacity at all times, MMC implemented systems to increase the number of weekend discharges, including utilizing throughput managers responsible for the discharge process. The results were dramatic: MMC's boarding decreased from 30 patients on average to almost zero within a year; LOS decreased; revenue increased; and a 30-bed inpatient unit was closed due to increased available capacity. ${ }^{45}$

\section{Full capacity action plan}

The previous three interventions serve to improve capacity, thus reducing boarding. However, economic factors require that hospitals run at near capacity; thus, small variations in flow can cause admitted patients to outpace the available inpatient beds. ${ }^{62,63}$ When capacity is exhausted, hospitals should utilize a program to handle excess admitted patients and reduce ED boarding, such as 
the full capacity protocol (FCP). The FCP redistributes boarders to inpatient units; these patients are placed in available areas of the inpatient floor (hallways, conference rooms, solarium, and exam room) instead of the ED hallway. The sickest patients requiring higher levels of care continue to board in the ED until an ICU or step-down bed is available. Institution of a FCP has been extensively studied in multiple settings and has been shown to decrease wait times, reduce boarding, improve throughput, shorten overall length of stay, and improve patient satisfaction..$^{64-68}$ In 2001, Stony Brook University Hospital developed a FCP in the face of ever-increasing ED boarding. During times of overcensus, a total of 9 units were asked to increase their census from 30 to 32 patients, allowing a total of 18 boarded patients to be redistributed throughout the hospital rather than left in the ED. Their protocol showed an improvement of approximately 4 hours per day in nursing hours per patient and a 0.8-day decrease in overall LOS. ${ }^{45,68}$ Additionally, a survey of patients who spent time boarding in ED and inpatient hallways, showed that patients preferred to board in the inpatient hallways. ${ }^{65} \mathrm{~A}$ full capacity protocol should be extensive enough to match the need, to assure that every patient's care and attention is maximized to the best of the institution's abilities. This is not possible if all boarders are simply left in the ED.

\section{CONCLUSION}

The term "ED overcrowding", when due to boarding of admitted patients, is more accurately termed "hospital overcrowding". Over the years, multiple small process improvement projects have attempted to improve ED overcrowding, including improving nursing report, rapid bed cleaning and bed turnaround, bedside registration, hospitalists in the ED, and increasing ED bed capacity. While important interventions, these processes do not improve the fundamental problem of improving hospital capacity. ${ }^{69} \mathrm{ED}$ boarding is a major patient safety issue, with studies demonstrating, increased LOS, increased medical errors, increased number of patients leaving without being seen and, increased mortality in both 10- and 30-day studies. ${ }^{34-36}$

With so many grave consequences, hospitals need to attack hospital overcrowding with interventions that have been shown to be effective. Four critical actions have been identified that, when effectively implemented to a sufficient degree, significantly decrease the number of admitted patients boarding within the ED and improve all associated metrics. These critical actions include the smoothing of elective admissions, implementation of both early daily discharges and continued discharges through the weekend, all to improve capacity, as well as the development and activation of the full capacity protocol when overall hospital ca- pacity has been exceeded. Targeting these four interventions means a focus on process improvements hospital-wide. Communication, cooperation, and compromise between the ED, inpatient and surgical services, and administrative leadership will be crucial for implementation. Change is challenging, but the demonstrated benefit through case studies show they can substantially reduce or eliminate boarding of admitted patients, enhance patient safety, improve capacity, and improve the financial health of the institution.

\section{CONFLICT OF INTEREST}

No potential conflict of interest relevant to this article was reported.

\section{REFERENCES}

1. American College of Emergency Physicians. Policy statement: crowding [Internet]. Irving, TX: American College of Emergency Physicians; 2013 [cited 2018 Jun 11]. Available from: https://www.acep.org/patient-care/policy-statements/crowdi ng/\#sm.000uy2fqlzc4ed610br2m5bif3m23.

2. Richardson DB, Mountain D. Myths versus facts in emergency department overcrowding and hospital access block. Med J Aust 2009;190:369-74.

3. Viccellio P. Emergency department overcrowding: an action plan. Acad Emerg Med 2001;8:185-7.

4. US Department of Health \& Human Services. National hospital ambulatory medical care survey: 2012 emergency department summary tables [Internet]. Atlanta, GA; Centers for Disease Control and Prevention; 2013 [cited 2018 Jun 11]. Available from https://www.cdc.gov/nchs/data/ahcd/nhamcs_ emergency/2012_ed_web_tables.pdf.

5. National Emergency Medical Center. 2012 Yearbook of emergency medical statistics [Internet]. Seoul: National Emergency Medical Center; 2013 [cited 2018 Jun 11]. Available from: http://www.nemc.or.kr.

6. QuickStats: percentage of emergency department visits with waiting time for a physician of $>1$ hour, by race/ethnicity and triage level: United States, 2003-2004. MMWR Morb Mortal Wkly Rep 2006;55;463.

7. Pines JM, Pollack CV Jr, Diercks DB, Chang AM, Shofer FS, Hollander JE. The association between emergency department crowding and adverse cardiovascular outcomes in patients with chest pain. Acad Emerg Med 2009;16:617-25.

8. Jo $\mathrm{S}$, Kim $\mathrm{K}$, Lee JH, et al. Emergency department crowding is associated with 28-day mortality in community-acquired 
pneumonia patients. J Infect 2012;64:268-75.

9. Bazarian JJ, Schneider SM, Newman VJ, Chodosh J. Do admitted patients held in the emergency department impact the throughput of treat-and-release patients? Acad Emerg Med 1996;3:1113-8.

10. Krall SP, Cornelius AP, Addison JB. Hospital factors impact variation in emergency department length of stay more than physician factors. West J Emerg Med 2014;15:158-64.

11. McCarthy ML, Zeger SL, Ding $R$, et al. Crowding delays treatment and lengthens emergency department length of stay, even among high-acuity patients. Ann Emerg Med 2009;54: 492-503.

12. White BA, Biddinger PD, Chang $Y$, Grabowski $B$, Carignan $S$, Brown DF. Boarding inpatients in the emergency department increases discharged patient length of stay. J Emerg Med 2013;44:230-5.

13. Ye L, Zhou G, He X, Shen W, Gan J, Zhang M. Prolonged length of stay in the emergency department in high-acuity patients at a Chinese tertiary hospital. Emerg Med Australas 2012;24:63440.

14. Krochmal P, Riley TA. Increased health care costs associated with ED overcrowding. Am J Emerg Med 1994;12:265-6.

15. Liew D, Liew D, Kennedy MP. Emergency department length of stay independently predicts excess inpatient length of stay. Med J Aust 2003;179:524-6.

16. Richardson DB. The access-block effect: relationship between delay to reaching an inpatient bed and inpatient length of stay. Med J Aust 2002;177:492-5.

17. Bekmezian A, Chung PJ. Boarding admitted children in the emergency department impacts inpatient outcomes. Pediatr Emerg Care 2012;28:236-42.

18. Huang 0 , Thind A, Dreyer JF, Zaric GS. The impact of delays to admission from the emergency department on inpatient outcomes. BMC Emerg Med 2010;10:16.

19. Weiss SJ, Ernst AA, Derlet R, King R, Bair A, Nick TG. Relationship between the National ED Overcrowding Scale and the number of patients who leave without being seen in an academic ED. Am J Emerg Med 2005;23:288-94.

20. Richardson DB, Bryant M. Confirmation of association between overcrowding and adverse events in patients who do not wait to be seen. Acad Emerg Med 2004;11:462.

21. Weissman JS, Rothschild JM, Bendavid E, et al. Hospital workload and adverse events. Med Care 2007;45:448-55.

22. Liu SW, Thomas SH, Gordon JA, Hamedani AG, Weissman JS. A pilot study examining undesirable events among emergency department-boarded patients awaiting inpatient beds. Ann Emerg Med 2009;54:381-5.
23. Kulstad EB, Sikka R, Sweis RT, Kelley KM, Rzechula KH. ED overcrowding is associated with an increased frequency of medication errors. Am J Emerg Med 2010;28:304-9.

24. Sills MR, Fairclough D, Ranade D, Kahn MG. Emergency department crowding is associated with decreased quality of care for children with acute asthma. Ann Emerg Med 2011; 57:191-200.

25. Bernstein SL, Aronsky D, Duseja $R$, et al. The effect of emergency department crowding on clinically oriented outcomes. Acad Emerg Med 2009;16:1-10.

26. Pines JM, Localio AR, Hollander JE, et al. The impact of emergency department crowding measures on time to antibiotics for patients with community-acquired pneumonia. Ann Emerg Med 2007;50:510-6.

27. Fee C, Weber EJ, Maak CA, Bacchetti P. Effect of emergency department crowding on time to antibiotics in patients admitted with community-acquired pneumonia. Ann Emerg Med 2007;50:501-9.

28. Hwang U, Richardson L, Livote E, Harris B, Spencer N, Sean Morrison R. Emergency department crowding and decreased quality of pain care. Acad Emerg Med 2008;15:1248-55.

29. Pines JM, Hollander JE. Emergency department crowding is associated with poor care for patients with severe pain. Ann Emerg Med 2008;51:1-5.

30. Falvo T, Grove L, Stachura R, Zirkin W. The financial impact of ambulance diversions and patient elopements. Acad Emerg Med 2007;14:58-62.

31. Handel DA, John McConnell K. The financial impact of ambulance diversion on inpatient hospital revenues and profits. Acad Emerg Med 2009;16:29-33.

32. McConnell KJ, Richards CF, Daya M, Weathers CC, Lowe RA. Ambulance diversion and lost hospital revenues. Ann Emerg Med 2006;48:702-10.

33. Pines JM, Heckman JD. Emergency department boarding and profit maximization for high-capacity hospitals: challenging conventional wisdom. Ann Emerg Med 2009;53:256-8.

34. McCusker J, Vadeboncoeur A, Lévesque JF, Ciampi A, Belzile E. Increases in emergency department occupancy are associated with adverse 30-day outcomes. Acad Emerg Med 2014;21: 1092-100.

35. Sprivulis PC, Da Silva JA, Jacobs IG, Frazer AR, Jelinek GA. The association between hospital overcrowding and mortality among patients admitted via Western Australian emergency departments. Med J Aust 2006;184:208-12.

36. Richardson DB. Increase in patient mortality at 10 days associated with emergency department overcrowding. Med J Aust 2006;184:213-6. 
37. Boden DG, Agarwal $A$, Hussain $T$, et al. Lowering levels of bed occupancy is associated with decreased inhospital mortality and improved performance on the 4-hour target in a UK District General Hospital. Emerg Med J 2016;33:85-90.

38. Geelhoed GC, de Klerk NH. Emergency department overcrowding, mortality and the 4-hour rule in Western Australia. Med J Aust 2012;196:122-6.

39. Honigman LS, Wiler JL, Rooks S, Ginde AA. National study of non-urgent emergency department visits and associated resource utilization. West J Emerg Med 2013;14:609-16.

40. Durand AC, Gentile S, Devictor B, et al. ED patients: how nonurgent are they? Systematic review of the emergency medicine literature. Am J Emerg Med 2011;29:333-45.

41. Sayah A, Lai-Becker M, Kingsley-Rocker $L$, Scott-Long $T$, O'Connor K, Lobon LF. Emergency Department expansion versus patient flow improvement: impact on patient experience of care. J Emerg Med 2016;50:339-48.

42. Khare RK, Powell ES, Reinhardt G, Lucenti M. Adding more beds to the emergency department or reducing admitted patient boarding times: which has a more significant influence on emergency department congestion? Ann Emerg Med 2009;53:575-85.

43. Han JH, Zhou C, France DJ, et al. The effect of emergency department expansion on emergency department overcrowding. Acad Emerg Med 2007;14:338-43.

44. Killeen J, Chan T, Castillo E, Jones K, Guss D. Inpatient nurse staffing to care for ED boarders does not relieve emergency department crowding. Acad Emerg Med 2007;14(5 Suppl): S82-3.

45. Viccellio P, Hochman KA, Semczuk P, Santora C. Right focus, right solution: how reducing variability in admission and discharge improves hospital capacity and flow. In: Litvak $E_{1}$ editor. Optimizing patient flow: advanced strategies for managing variability to enhance access, quality, and safety. Oak Brook, IL: The Joint Commission; 2018. p.97-112.

46. Asplin BR, Flottemesch TJ, Gordon BD. Developing models for patient flow and daily surge capacity research. Acad Emerg Med 2006;13:1109-13.

47. Marcilio I, Hajat S, Gouveia N. Forecasting daily emergency department visits using calendar variables and ambient temperature readings. Acad Emerg Med 2013;20:769-77.

48. Boyle J, Jessup M, Crilly J, et al. Predicting emergency department admissions. Emerg Med J 2012;29:358-65.

49. Fieldston ES, Ragavan $M$, Jayaraman $B$, Allebach $K$, Pati S, Metlay JP. Scheduled admissions and high occupancy at a children's hospital. J Hosp Med 2011;6:81-7.

50. Ragavan MV, Blinman TA, Fieldston ES. Scheduled surgery admissions and occupancy at a children's hospital: variation we can control to improve efficiency and value in health care delivery. Ann Surg 2013;257:564-70.

51. McManus ML, Long MC, Cooper $A$, et al. Variability in surgical caseload and access to intensive care services. Anesthesiology 2003;98:1491-6.

52. Dempsey CJ. Managing variability in perioperative services. AORN J 2009;90:677-94.

53. Singh D, Terwiesch C. Benefits of surgical smoothing and spare capacity: an econometric analysis of patient flow. Prod Oper Manag 2017;26:1663-84.

54. Litvak E, Fineberg HV. Smoothing the way to high quality, safety, and economy. N Engl J Med 2013;369:1581-3.

55. Boston hospital sees big impact from smoothing elective schedule. OR Manager 2004;20:10-2.

56. Powell ES, Khare RK, Venkatesh AK, Van Roo BD, Adams JG, Reinhardt $\mathrm{G}$. The relationship between inpatient discharge timing and emergency department boarding. J Emerg Med 2012;42:186-96.

57. Beck MJ, Okerblom D, Kumar A, Bandyopadhyay S, Scalzi LV. Lean intervention improves patient discharge times, improves emergency department throughput and reduces congestion. Hosp Pract (1995) 2016;44:252-9.

58. Hayes R. Better discharge process cuts ED length of stay [Internet]. Waukesha, WI: GE Healhcare; 2009 [cited 2018 Jun 11]. Available from: http://www3.gehealthcare.com/ /media/ Downloads/us/Services/Performance\%20Solutions/CapacityManagement/GEHealthcare-Case-Study_Better_Discharge_ Process_Cuts_ED_Length_Apr09.pdf.

59. Wertheimer $B$, Jacobs RE, Bailey $M$, et al. Discharge before noon: an achievable hospital goal. J Hosp Med 2014;9:210-4.

60. Wertheimer $B$, Jacobs RE, Iturrate $E_{1}$ Bailey $M$, Hochman K. Discharge before noon: effect on throughput and sustainability. J Hosp Med 2015;10:664-9.

61. Wong HJ, Wu RC, Caesar M, Abrams H, Morra D. Smoothing inpatient discharges decreases emergency department congestion: a system dynamics simulation model. Emerg Med J 2010;27:593-8.

62. Pauly MV, Wilson P. Hospital output forecasts and the cost of empty hospital beds. Health Serv Res 1986;21:403-28.

63. Greene J. Emergency department flow and the boarded patient: how to get admitted patients upstairs. Ann Emerg Med 2007;49:68-70.

64. Garson C, Hollander JE, Rhodes KV, Shofer FS, Baxt WG, Pines JM. Emergency department patient preferences for boarding locations when hospitals are at full capacity. Ann Emerg Med 2008;51:9-12. 
65. Viccellio $P$, Zito JA, Sayage $V$, et al. Patients overwhelmingly prefer inpatient boarding to emergency department boarding. J Emerg Med 2013;45:942-6.

66. Willard E, Carlton EF, Moffat L, Barth BE. A full-capacity protocol allows for increased emergency patient volume and hospital admissions. J Emerg Nurs 2017;43:413-8.

67. Boyle $A$, Viccellio $P$, Whale $C$. Is "boarding" appropriate to help reduce crowding in emergency departments? BMJ 2015; $350:$ h2249.
68. Viccellio A, Santora C, Singer AJ, Thode HC Jr, Henry MC. The association between transfer of emergency department boarders to inpatient hallways and mortality: a 4-year experience. Ann Emerg Med 2009;54:487-91.

69. Salway RJ, Valenzuela R, Shoenberger JM, Mallon WK, Viccellio A. Emergency department (ED) overcrowding: evidencebased answers to frequently asked questions. Revista Medica Clinica Las Condes 2017;28:213-9. 